\title{
Point de vue médical concernant les troubles douloureux
}

\author{
Jörg Jeger \\ Dr med., spécialiste en rhumatologie FMH, EMBA, MAS en médecine des assurances, médecin-chef MEDAS Zentralschweiz
}

Dès les années quatre-vingt, les versements anticipés de rentes ont massivement augmenté. Malheureusement, les sociétés de discipline médicale ont tardé à se pencher sur cette importante question de politique sociale, si bien que le Tribunal fédéral a tranché en 2004 et mis un terme à cette pratique, confirmant ainsi un vieil adage de Swissair dans les années cinquante: «Qui ne vole pas sera survolé.»

\section{L'évaluation de la capacité de travail: une tâche négligée}

De toute évidence, la formation médicale néglige encore de façon inexcusable l'évaluation de la capacité de travail. C'est ce qu'a démontré un sondage réalisé auprès des médecins spécialistes récemment diplômés publié il y a peu dans cette revue [1]. En effet, parmi les 23 objectifs de formation faisant l'objet de ce sondage, l'évaluation de la capacité de travail a obtenu les moins bonnes évaluations dans tous les domaines spécialisés. Il existe donc un retard à combler impérativement dans le cadre de la formation pré- et postgraduée.

\section{Inconvénients liés à la jurisprudence} rendue jusqu'alors en ce qui concerne les troubles douloureux («Überwindbarkeitspraxis" ou présomption du caractère surmontable des troubles)

- La base de données empiriques sur laquelle repose la présomption du Tribunal fédéral selon laquelle les troubles somatoformes peuvent "être surmontés par un effort de volonté raisonnablement exigible» est très peu fournie et la référence à «l'empirie médicale» n'est pas défendable [2].

- Le regroupement des tableaux cliniques mentionnés sous la notion de "syndrome sans pathogenèse ni étiologie claires et sans constat de déficit organique» s'avère problématique. Il s'agit d'une notion juridique qui ne peut être transposée telle quelle en médecine.

- Le Tribunal fédéral a considérablement modifié les critères liés au pronostic négatif qu'avait définis le psychiatre tubingois Klaus Foerster et leur a attribué une portée différente. Il a notamment accordé à la comorbidité psychiatrique une importance qu'on ne saurait justifier d'un point de vue médical. Celle-ci a effectivement été érigée au rang de critère exclusif de la demande de rente.

- Cette jurisprudence a abouti, en règle générale, à des décisions de type "tout ou rien». Or, la situation médicale des patients présentant des troubles psychosomatiques implique souvent que ceux-ci disposent de ressources résiduelles dont ils pourraient faire un usage judicieux dans la vie active. Ces ressources ne sont toutefois pas suffisantes pour assumer un poste à plein temps sur le marché du travail.

- Le défaut d'objectivation n'est pas corrélé, en soi, avec «l'effort de volonté qu'on peut raisonnablement exiger pour surmonter» les troubles concernés.

- L'ancienne jurisprudence relative aux troubles douloureux a été conçue pour des diagnostics déterminés. Cela explique la conception erronée selon laquelle un diagnostic permettrait de tirer des conclusions sur le degré d'incapacité.

- L'ancienne pratique du caractère surmontable des troubles était difficile à communiquer aux assurés et menait à des formulations blessantes et dégradantes. Cette pratique a également fortement contribué au durcissement des positions entre organes de l'assurance-invalidité et médecins, un sujet qui a plusieurs fois été traité dans cette revue.

\section{Le tournant: l'arrêt 9C_492/2014 du 3 juin 2015}

L'essentiel en bref:

- La présomption du caractère surmontable de la douleur est abandonnée, faute de fondement scientifique suffisant. 
- Le Tribunal fédéral parle désormais de "syndromes somatoformes douloureux persistants et troubles psychosomatiques analogues».

- L'ancienne procédure, construite sur le modèle règle/ exception, est remplacée par une procédure probatoire structurée fondée sur des indicateurs prédéfinis. L'importance de la comorbidité psychiatrique est fortement relativisée et le critère du bénéfice primaire tiré de la maladie complètement abandonné.

- C'est à dessein que ces facteurs sont désignés comme des indicateurs, et non comme des critères. Chaque cas doit être examiné avec le même sérieux et sans a priori, indépendamment du diagnostic.

- En se fondant sur les indicateurs, les médecins traitants et les médecins qui procèdent à l'expertise doivent déterminer dans quelle mesure les troubles concernés induisent des limitations fonctionnelles ayant des répercussions sur l'activité et la participation. Les indicateurs concernent le degré de gravité fonctionnel de la maladie et la cohérence de l'incapacité (limitations identiques du niveau d'activité dans des domaines comparables de la vie, degré de souffrance).

- La nouvelle jurisprudence attache de l'importance au fait de prendre en compte et d'établir non seulement les déficiences (représentation négative des capacités), mais également les ressources (représentation positive des capacités). Cette nouvelle approche s'exprime dans la phrase: "L'incapacité de travail découle du solde de l'ensemble des handicaps et des ressources.» (traduction libre).

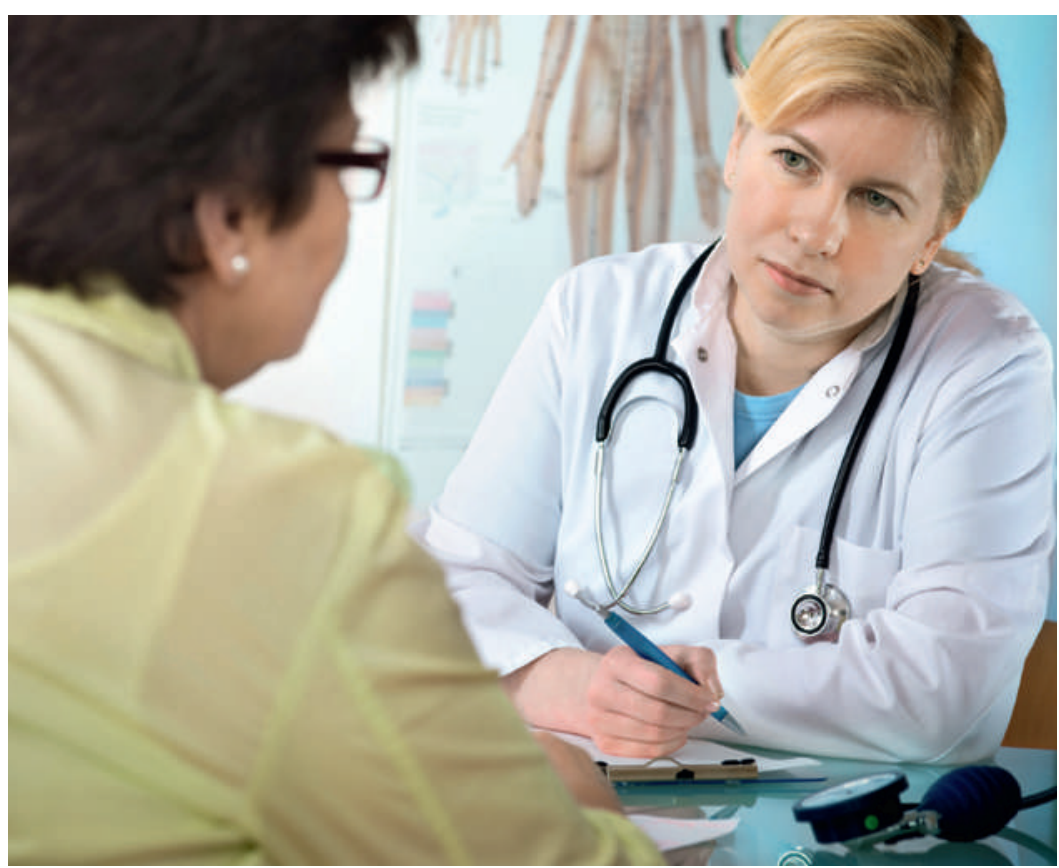

La nouvelle jurisprudence attache de l'importance au fait de prendre en compte et d'établir non seulement les déficiences, mais également les ressources.
- Le fardeau de la preuve continue d'incomber au requérant: si celui-ci ne parvient pas à établir la preuve cohérente de l'incapacité, aucune prestation d'assurance (aucune rente, en particulier) n'est due.

\section{Première appréciation du point de vue médical}

Le Tribunal fédéral s'est prononcé plus vite que prévu et de manière assez radicale sur les erreurs commises, auxquelles il a remédié. Avec la nouvelle jurisprudence relative aux symptômes cliniques psychosomatiques, il a franchi une étape importante qui ouvre des perspectives d'avenir et de modernité. En effet, il a remédié d'un seul coup à pratiquement toutes les lacunes rele-

\section{Le Tribunal fédéral s'est prononcé plus vite} que prévu et de manière assez radicale sur les erreurs commises.

vées durant les dix dernières années d'un point de vue médical et juridique. Ce faisant, le Tribunal fédéral a fait preuve d'une ouverture d'esprit et d'un sens de l'autocritique étonnants.

- Il faut y voir l'aveu que l'ancienne jurisprudence incitait à considérer la règle générale comme immuable et à la cristalliser. Le Tribunal fédéral parle à cet égard de «Bias» (erreur systématique), ce qui donne à réfléchir.

- La procédure probatoire structurée fondée sur des indicateurs ne préjuge pas de l'issue de la requête. Tant les médecins chargés de l'évaluation que les praticiens du droit doivent examiner avec la même diligence, et indépendamment du diagnostic, s'il y a ou non incapacité. Le fait qu'une telle incapacité doive être cohérente et analogue dans des domaines comparables de la vie doit aussi être souligné et soutenu du point de vue médical.

- Le praticien du droit exige en outre une appréciation objective de l'exigibilité. Cela signifie qu'il n'appartient pas seulement au sujet concerné d'établir que sa capacité de travail est réduite, mais que les divers spécialistes du domaine médical et de la réadaptation professionnelle doivent parvenir à la conclusion qu'il existe une réelle incapacité ayant des répercussions sur la vie professionnelle. Les observations des tentatives de réadaptation encadrées par des professionnels revêtent ainsi une plus grande portée.

- La plus grande modification, et à mes yeux la plus belle, réside dans la conception de l'être humain sur laquelle se fonde la nouvelle jurisprudence: toutes les personnes prenant part à la procédure doivent 
traiter le requérant avec la même diligence et le même respect, indépendamment du diagnostic. Il n'y a plus de «Geht-uns-sowieso-nichts-an-Diagnosen» (GUSNAD), et les décisions ou les jugements refusant une prestation ne devraient plus non plus contenir de formulations attentatoires à la personnalité.

- En ce qui concerne le diagnostic, la nouvelle jurisprudence met l'accent sur la preuve de l'incapacité (limitations fonctionnelles ayant des répercussions sur l'activité et la participation).

- L'arrêt concerné s'imprègne d'une approche moderne de la médecine et de la réadaptation: l'incapacité résulte de l'interaction entre l'individu, affecté

\section{Le Tribunal fédéral a également clairement précisé qu'il attendait des sociétés de discipline qu'elles édictent des directives relatives à} l'évaluation des troubles psychosomatiques.

par une maladie ou un accident, et son environnement. Le fait qu'un être humain puisse assumer ou non ses obligations sociales dépend du rapport entre ses limitations fonctionnelles (déficiences), ses ressources personnelles et les exigences de la vie.

- La nouvelle jurisprudence pose des conditions strictes tant aux médecins chargés de l'évaluation qu'aux praticiens du droit. Il reste à espérer que tous les intéressés soient en mesure de satisfaire à ces exigences. Le monde médical devra approfondir sa formation en matière d'évaluation de la capacité de travail ainsi que la mise en œuvre de la classification ICF. Les sociétés de discipline et la FMH seront particulièrement sollicitées à cet égard. Le Tribunal fédéral a également clairement précisé qu'il attendait des sociétés de discipline qu'elles édictent des directives relatives à l'évaluation des troubles psychosomatiques. Plus la jurisprudence pourra s'appuyer sur un consensus probant des associations professionnelles, moins il lui sera nécessaire de recourir à ses propres constructions juridiques auxiliaires. Les médecins doivent se le mettre en tête!

- Certaines incertitudes demeureront tant que le praticien du droit ne définira pas le seuil de la preuve de l'incapacité. Ce point relève d'un pouvoir d'appréciation exploité par les divers intervenants.

- Il ne faut en aucun cas se méprendre sur la nouvelle jurisprudence et considérer qu'elle ouvre la porte à une allocation libérale des rentes comme dans les années nonante. On ne le soulignera jamais assez: est exigée la preuve cohérente de l'incapacité, ni plus, ni moins. Le monde médical doit tirer les enseignements du passé. S'il ne le fait pas, il sera assurément laissé pour compte une prochaine fois (et probablement de manière définitive). Nous devons faire un usage diligent et prudent des responsabilités que nous avons recouvrées!

- Dans une Jusletter récemment publiée [3], les juristes Thomas Gächter et Michael Meier ont parlé de "signes d'une culture judiciaire élevée». Je ne peux qu'adhérer de manière inconditionnelle à leurs propos. En tant que médecin, mais aussi en tant que citoyen, j'aimerais saluer cette avancée et l'accueillir avec des remerciements sincères. C'est un arrêt moderne qui tient largement compte des progrès de la médecine et de la réadaptation.

Références

1 Siegrist $M$ et al. Rétrospective de la formation postgraduée de médecin spécialiste. Bull Méd Suisses. 2015; 96(22):758-63.

2 Jeger J. Die Entwicklung der «FOERSTER-Kriterien» und ihre Übernahme in die bundesgerichtliche Rechtsprechung: Geschichte einer Evidenz. Jusletter. 16 mai 2011. (www.jusletter.ch).

3 Gächter T, Meier ME. Schmerzrechtsprechung 2.O. Jusletter. 29 juin 2015. (www.jusletter.ch).

Credit photo

Alexander Raths | Dreamstime.com 